\title{
Design of a customised bridging mandibular prosthesis for complex reconstruction: a pilot study
}

\author{
Realizzazione di una protesi personalizzata per le ricostruzioni complesse della \\ mandibola: studio pilota
}

\author{
A. TARSITANO 1 , S. BATTAGLIA ${ }^{1}$, A. SANDI ${ }^{2}$, C. MARCHETTI ${ }^{1}$ \\ ${ }^{1}$ Maxillofacial Surgery Unit, S. Orsola-Malpighi Hospital, Department of Biomedical and Neuromotor Sciences, Alma \\ Mater Studiorum University of Bologna, Italy; ${ }^{2}$ SINTAC s.r.l. Biomedical Engineering, Trento, Italy
}

\section{SUMMARY}

The gold standard for mandibular reconstruction is universally recognised and consists of the replacement of the bony part of the mandible with a bony microvascular free flap supported by a reconstructive plate. Although this procedure is feasible and reproducible in most patients, at times poor oncological prognosis or poor performance status force surgeons to consider other reconstructive solutions. In these cases, the main alternative in reconstructing a mandibular defect is represented by bridging plates combined with soft tissue flaps. However, repairing a mandibular defect with a reconstructive plate only can lead to a series of diverse complications. The most frequent complications reported are rupture and oral exposure of the plate. In this paper, we describe a new method for mandibular reconstruction using a customised bridging mandibular prosthesis (CBMP) without bone free flap.

KEY WORDS: Computer-aided design $\bullet$ Computer-aided manufacturing $\bullet$ Mandibular reconstruction $\bullet$ Reconstructive surgery $\bullet$ Bridging plate $\cdot$ Prosthesis

\section{RIASSUNTO}

La ricostruzione mandibolare è attualmente effettuata mediante il trasferimento di lembi liberi rivascolarizzati di tessuto osseo, supportati da placche di osteosintesi. Sebbene questa procedura sia generalmente efficace e riproducibile, talvolta la scarsa prognosi oncologica o le condizioni cliniche scadenti del paziente costringono il chirurgo a considerare alternative ricostruttive. In tali casi, la principale possibilità è rappresentata dall'utilizzo di placche ricostruttive 'a ponte', associate a lembi liberi di tessuti molli. Comunque la ricostruzione così concepita espone a un significativo rischio di sviluppare complicanze di vario genere. Le più frequenti complicanze sono rappresentate dalla rottura e dall'esposizione della placca. In questo articolo descriviamo un nuovo metodo ricostruttivo mandibolare, che si avvale di una protesi mandibolare customizzata, senza lembo libero osseo.

PAROLE CHIAVE: Progettazione computer-assistita $\bullet$ Costruzione computer-assistita $・$ Ricostruzione mandibolare $\cdot$ Chirurgia ricostruttiva $\bullet$ Placca a ponte $\bullet$ Protesi

Acta Otorhinolaryngol Ital 2017;37:195-200

\section{Introduction}

Mandibular reconstruction after wide bone resections is a well-standardised procedure. The gold standard for mandibular reconstruction is universally recognised and consists of the replacement of the bony part of the mandible with a bony microvascular free flap supported by a reconstructive plate ${ }^{1-3}$.

Although this procedure is feasible and reproducible in most patients, at times poor oncological prognosis or poor performance status together with other relative or absolute vascular contraindications force surgeons to consider other reconstructive solutions ${ }^{4-6}$. In these cases, the main alternative in reconstructing a mandibular defect is represented by bridging plates combined with soft tissue flaps, especially when the condyles are bilaterally preserved ${ }^{7-9}$. Considering biomechanical and structural results, this procedure guarantees a reasonable alternative to a bony free flap reconstruction ${ }^{4}$.

However, repairing a mandibular defect with a reconstructive plate only can lead to a series of diverse complications. The most frequent complications are rupture and oral exposure of the plate ${ }^{1011}$. The latter complication is mostly due to the misplacement of plate positioning during the surgical reconstructive procedure. In both cases, the plate has to be removed, creating a challenging surgical scenario for the surgeon. In fact, after the removal of a mandibular plate, a secondary approach and mandibular reconstruction is always difficult due to the lack of healthy bone on which to fix the new plate.

In this paper, we describe a new method for mandibular reconstruction using a customised bridging mandibular prosthesis (CBMP) without bone free flap. 


\section{Clinical techniques and technologies}

A 70-year-old patient had the following clinical history: onset of mandibular pain and swelling since 2011. The symptoms persisted after therapy with antibiotics and painkillers. Four years earlier the patient had been surgically treated for prostate adenocarcinoma.

Afterwards, he developed multiple bone metastases. These were treated with chemotherapy associated with zolendronic acid. The patient clinically presented multiple skin and oral fistulas of the anterior region of the mandible and a large bisphosphonate-related osteonecrosis of the mandibular bone.

Since he was affected by multiple bone metastases (lower leg, ankle, spine), fibular and DCIA free flaps reconstruction was not appropriate. Therefore, the procedure chosen for the correction of the defect, after mandibular segmental resection, was the use of a reconstructive plate and a soft tissue microvascular free flap (anterolateral thigh flap, ALTF).

Ten days after surgery, plate exposure appeared in the anterior vestibular oral region of the mouth. The exposure became larger, exposing all the anterior sector of the plate (Fig. 1). Furthermore, the lack of soft tissue anchors allowed the mouth to posteriorly and inferiorly collapse.

When the patient came to our Department, four months after the previous surgery, we identified bilateral rami anti-version and rotation without condyle displacement (Fig. 2). This was probably caused by an intra-operative misplacement of the reconstructive plate, which was partially responsible for its oral exposure.

Therefore, 5 months after the first surgery, we decided to replace the reconstructive plate with a customised bridging mandibular prosthesis (CBMP).

Virtual planning began by processing Digital Imaging and Communications in Medicine (DICOM) files from CT scan data of the patient, using Rhino software, version 4.0 (Robert McNeel \& Associates, Seattle, WA, USA).

Superimposition of pre-resection CT scan and post-resection CT scan of the mandible was performed according to a best-fit algorithm using 3-Matic software (Materialise, Leuven, Belgium). This demonstrated the displacement of both of the mandible rami (Fig. 3). The virtual project
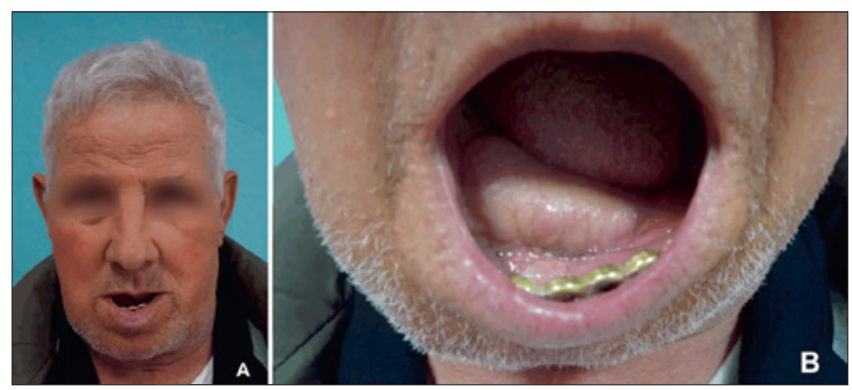

Fig. 1. Clinical view of reconstructive plate exposure. A: facial appearance. B: intra-oral exposure.

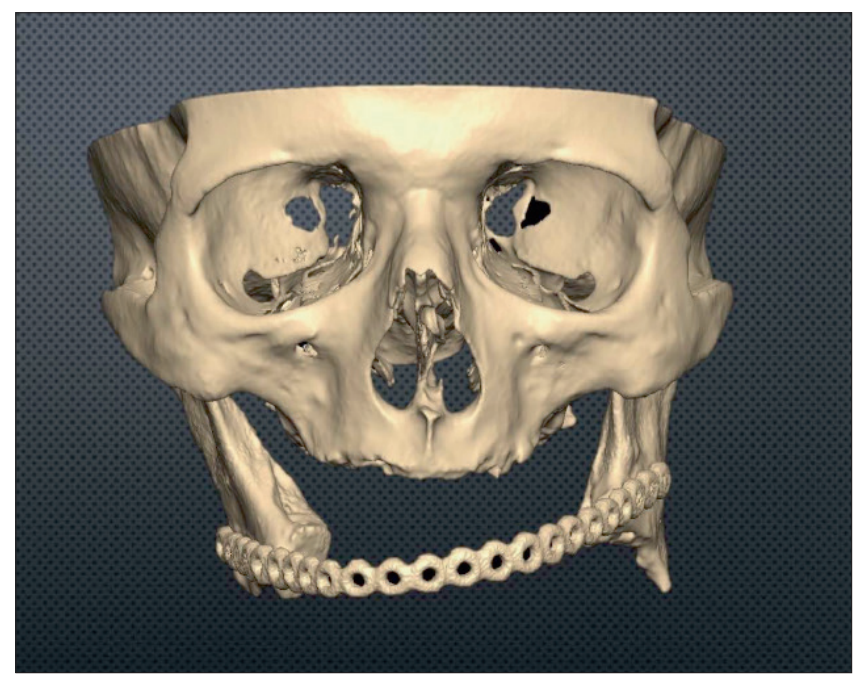

Fig. 2. 3D model of CT showing bilateral rami anti-version and rotation without condyle displacement.

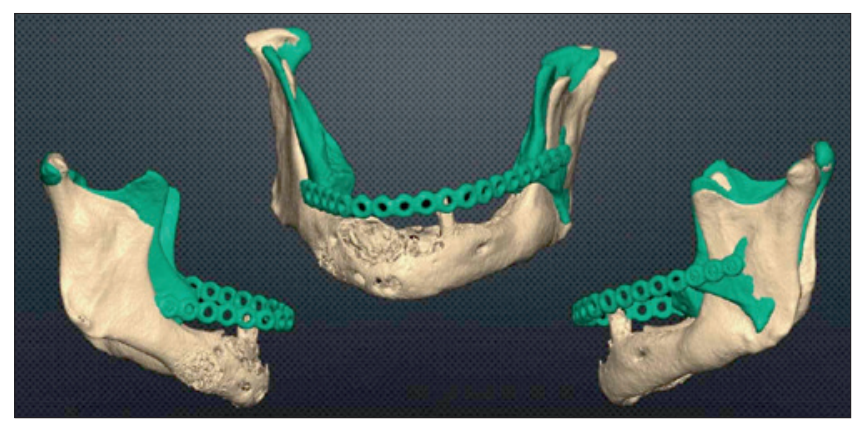

Fig. 3. Superimposition of native mandible (yellow) and post-resection (green) CT-scan data showing mandibular rami displacement.

was carried out with a biomedical engineer (SINTAC s.r.l. Biomedical Engineering, Trento, Italy), realising a CBMP to reach our surgical goal.

The virtual planning consists in the mandibular rami repositioning as well as in the pre-resection situation. Afterwards, a CBMP was designed in order to reconstruct the anterior part of the mandible and restore mandibular continuity. CBMP was designed on the internal (lingual) cortical bone surface of the native mandible in order to reduce the tension on soft tissues of the chin region (Fig. 4). CBMP was designed as a mandible-like bridging prosthesis, with a rounded three-dimensional surface in order to reduce the damage at prosthesis/soft tissues interface. The height of the CBMP was $1 \mathrm{~cm}$ and the minimum thickness was $3 \mathrm{~mm}$. Two retention titanium structures were designed on each plate-end side to increase hardware stability (Fig. 5).

Twelve holes were provided on the anterior part, corresponding to the chin. These should allow the surgeon to position the anterior digastric and genioglossus muscles on the prosthetic chin. The part of the prosthesis corresponding to the mandibular body has some grooves in order to fix the mylohyoid muscles (Fig. 5). Repositioning 


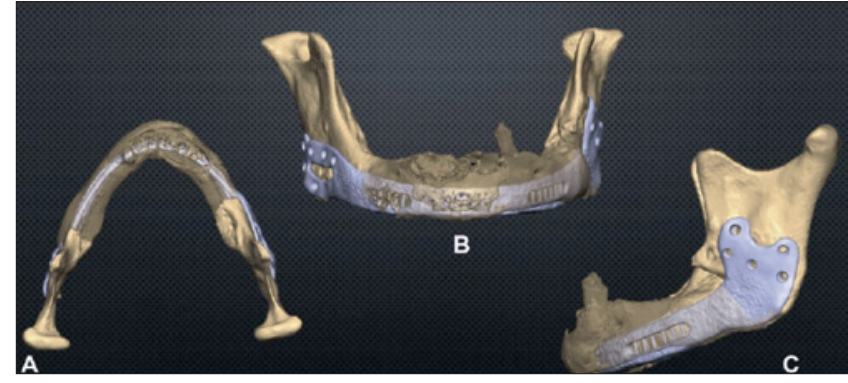

Fig. 4. Computer-assisted design of CBMP. CBMP is designed on the internal (lingual) bone surface of the native mandible. A: inferior view. B: anterior view. C: left view.

the suprahyoid muscles on the CBMP should both avoid the antero-rotation of the mandibular rami and restore more physiological mandibular movements.

To achieve accurate CBMP positioning, we designed two drilling guides (Fig. 6). Their design was made to fit on the previously placed reconstructive plate. This allowed the reduction of potential errors during intra-operative positioning. Drilling guides were used to perform the new screw holes and, since the holes for the fixing screws of the guides were the same as those in the CBMP, to obtain the correct positioning of CBMP and, consequently, of the mandibular rami. The guides were of polyamide and manufactured using a 3-D Printer.

The CBMP was manufactured by a direct metal laser sintering method: titanium Ti64 was fused into a solid component and melted locally using a focused laser beam ${ }^{12}$.

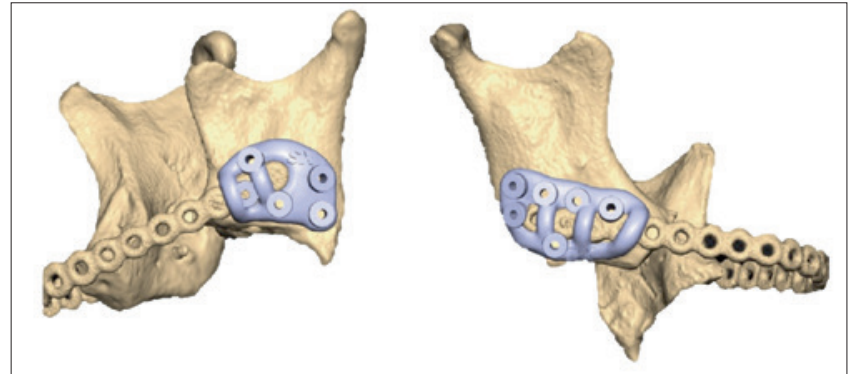

Fig. 6. Computer-assisted design of drilling guides: they were designed to fit on the previously placed reconstructive plate.

The solid-to-layer files of the guide and plate were then manufactured through direct metal laser sintering (DMLS) using an EOSINT M270 system (Electro-Optical Systems, GmbH, Munich, Germany). DMLS was used to fuse the titanium powder into a solid form and then melt it locally with a focused laser beam. As with other additive manufacturing technologies, the components were built up additively in layers.

Cervical surgical access was performed. Oral access was avoided in order to reduce the risk of further future plate exposure, since the oral mucosa and the soft tissue of the ALTF conditions were in good healing status.

Drilling guides were positioned on the pre-planned sites and fixed with screws (Fig. 7).

Then, the reconstructive plate was removed and substituted with the CBMP. Mandibular prosthesis was placed

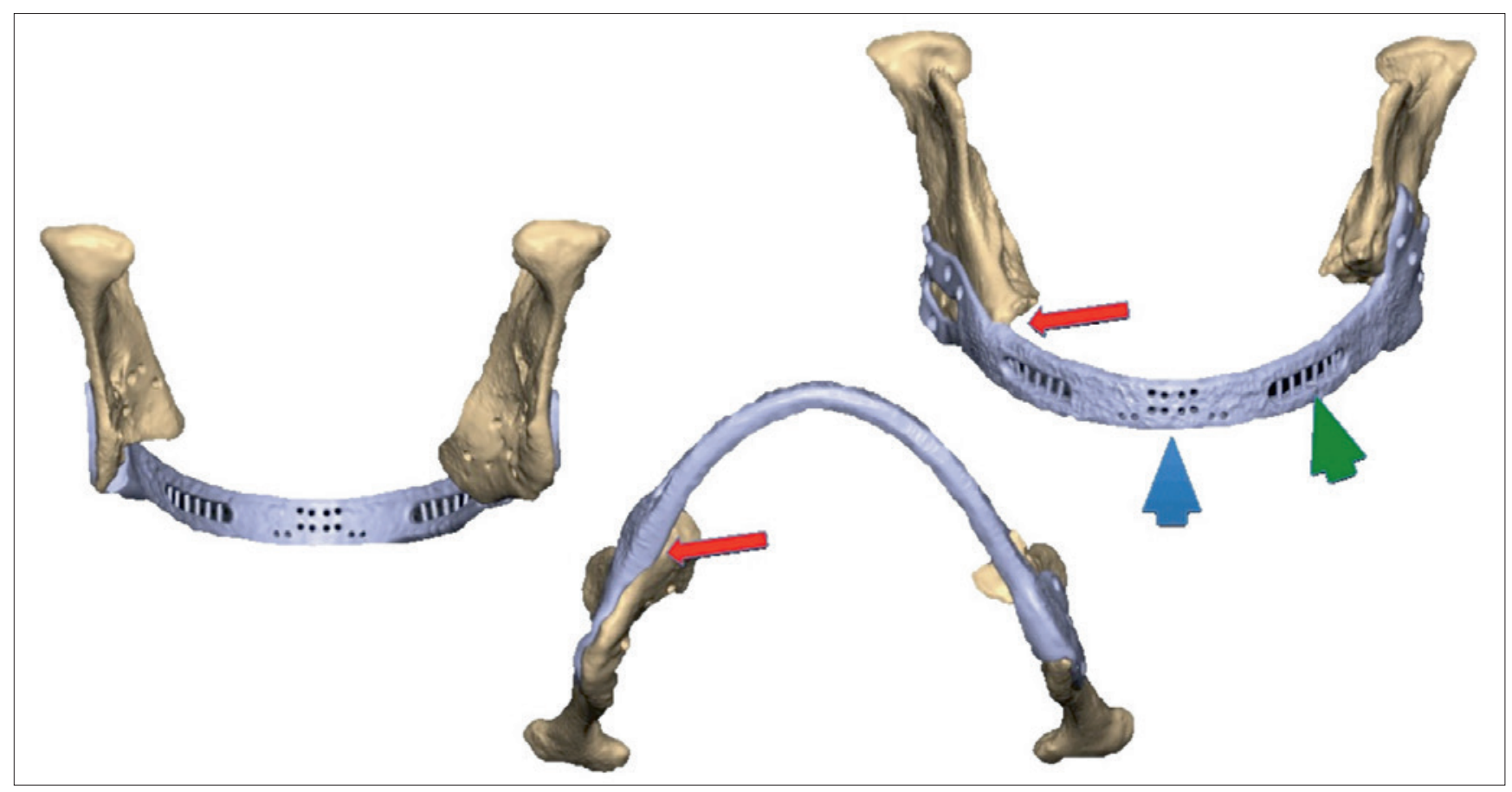

Fig. 5. Computer-assisted design of CBMP. Two retention titanium structures were designed on each plate-end side in order to increase hardware stability (red arrow). Twelve holes were provided on the anterior part (blue arrow). These should allow the surgeon to position the anterior digastric and genioglossus muscles on the prosthetic chin. The part of the prosthesis corresponding to the mandibular body has some grooves in order to fix the mylohyoid muscles (green arrow). 

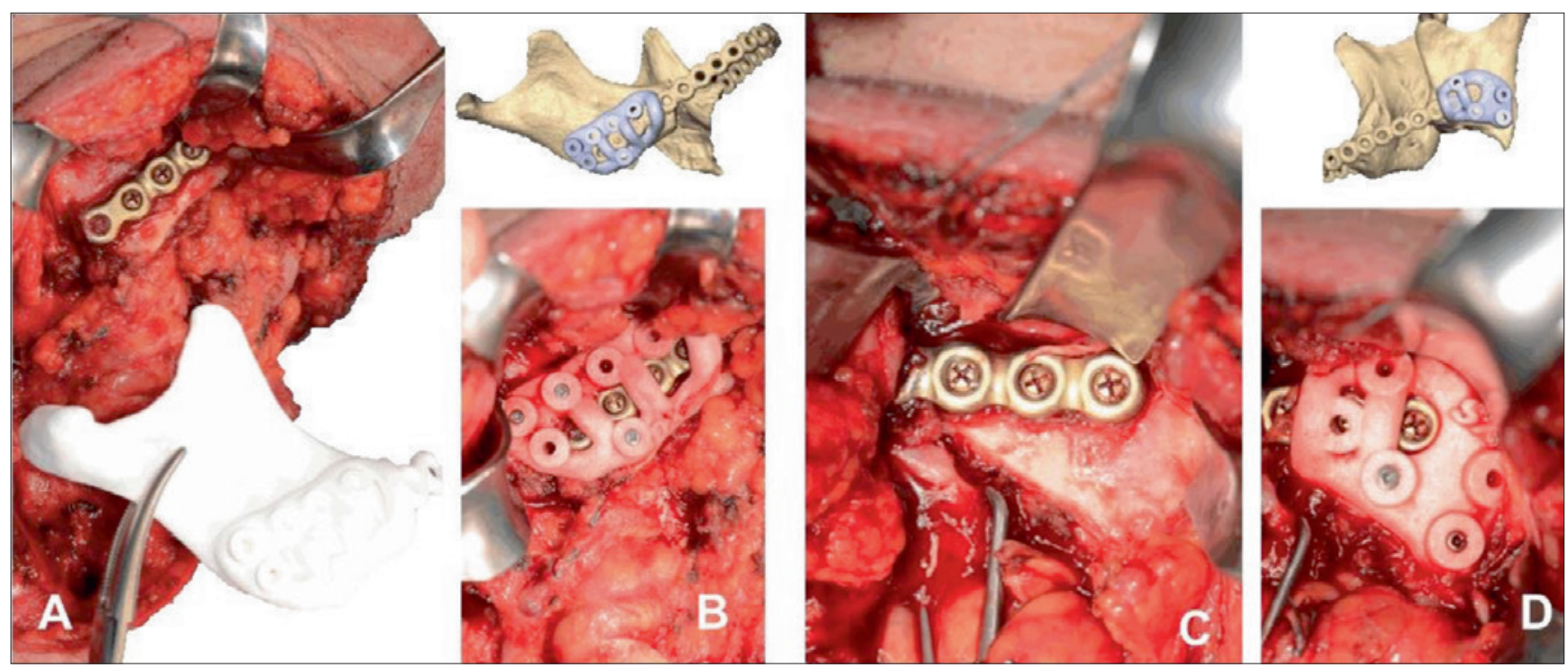

Fig. 7. Intra-operative image showing drilling guides positioning. A: right plate side exposition; B: right drilling guide positioning on the plate; C: left plate side exposition; D: left drilling guide positioning on the plate.
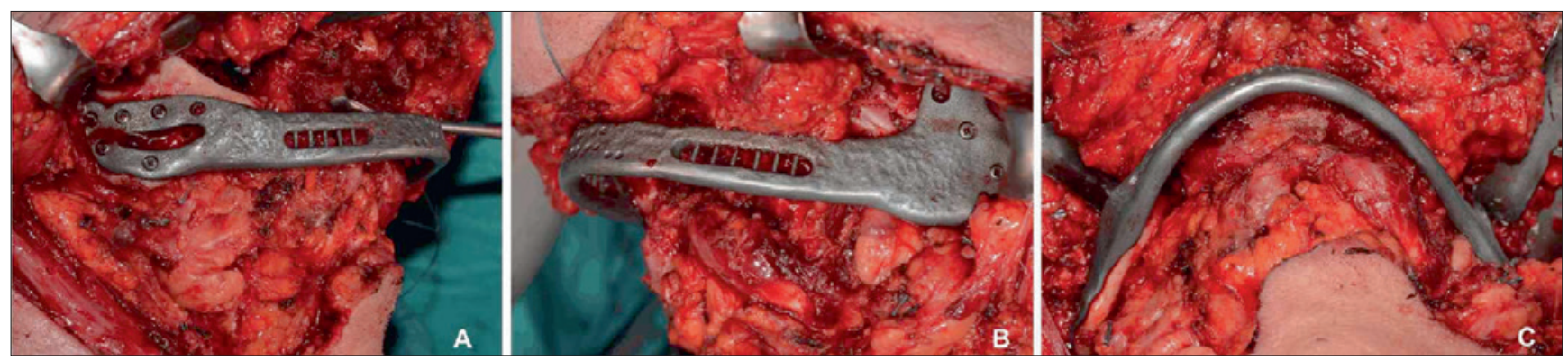

Fig. 8. Intra-operative images showing CBMP positioning. Note the nice fitting of the customised implant to the mandible. A: right view; B: left view; C: inferior view.

by using holes performed following drilling guides and fixed to the bone with 2.4 bicortical screws. The CBMP perfectly fit in the planned position (Fig. 8).

Suprahyoid muscles were anchored to the anterior part of the CBMP using non-resorbable sutures, as well as mylohyoid muscles in the lateral portion of the prosthesis.

The post-operative course was uneventful and the patient was discharged without complications. No radiological signs of CBMP dislocation were identified in post-operative $\mathrm{CT}$ scan.

At 3 months follow-up, no oral exposure of CBMP was detected (Fig. 9).

A post-operative CT scan was performed 1 week after surgery to confirm the accuracy of the reconstruction. Morphological results were evaluated using SimPlant O\&O software (Dentsply Implants, Mölndal, Sweden). The difference between the virtually-planned and postoperative position of the CBMP was then calculated. The pre-operative $\mathrm{CT}$ data set used for virtual planning was superimposed onto the postoperative CT scan, while the "best fit" algorithm of the GOM (GOM mbH, Braunsch- weig, Germany) 3D software enabled overlapping of the skull to provide the exact points of deviation. These were obtained by determining the deviation between the preoperative design and post-operative outcome. First, 3D rendering of the bone contour was reconstructed from the post-operative CT scan and exported into the STL

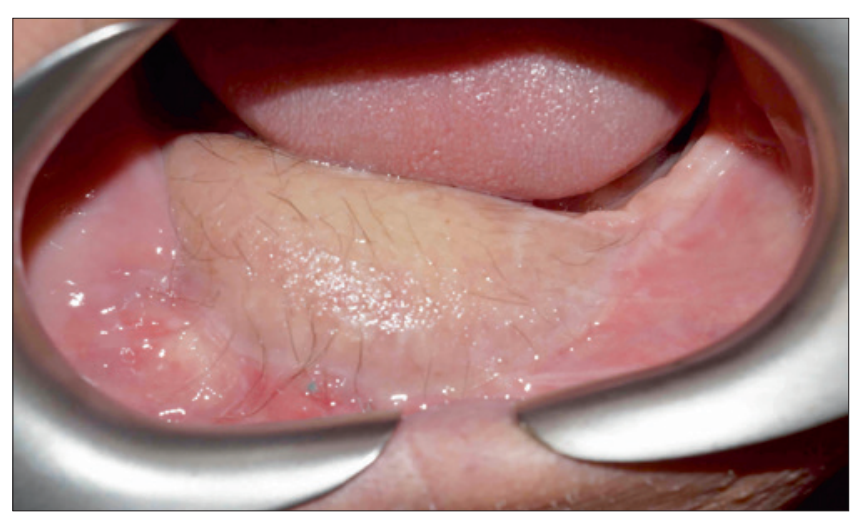

Fig. 9. Intra-oral image showing good soft tissue healing without signs of implant exposure. 
files. Second, the pre- and post-operative data were imported into the software using manual and global registration functions to match the non-surgical parts of the two models. A 3D comparison was then performed and the deviation from the pre-operative design was reflected in a deviation spectrum. The program is able to recognise corresponding points from two images and highlight them on the superimposed image using different colours according to the distance between corresponding points. The resulting error grade colour map provides a direct impression of the concordance between the pre-operative design and the post-operative outcome (Fig. 10).

The error grade colour map revealed that good reproducibility was obtained with an average error of $0.4 \mathrm{~mm}$.

In particular, the area of the condyle presented an average error between 0 and $0.4 \mathrm{~mm}$, compared to pre-resection CT-scan; the mandible angle presented an average error ranging between 0 and $0.8 \mathrm{~mm}$ and the coronoid a mean error of $1.30 \mathrm{~mm}$ (Fig. 10).

\section{Discussion}

The state-of-the-art treatment for anterior mandibular defects is primary bone reconstruction, which prevents major facial deformities, provides the lip support that is essential for labial competence, and restores bone for osteointegrated implants ${ }^{4}$.

In fact, anterior mandibular arch resections always cause major aesthetic and functional problems: loss of the anterior tongue attachments causes retroposition of the oral cavity soft tissues and suprahyoid muscles, which gives rise to a reduction of airway volume and the loss of oral competence with continuous drooling. Whereas loss of the symphysis and segments of the mandibular body may be so detrimental that the patient becomes a social recluse, determining an Andy-Gump deformity. Consequently, there is an absolute need for reconstruction techniques that not only restore mandibular continuity, but also replace suprahyoid muscles. Reconstruction with bridging plates is an alternative surgical option if microvascular reconstruction using bone free flap is not possible ${ }^{4}$. Although reconstructive plates fixed to the mandibular stumps restore bone continuity and maintain residual occlusion and TMJ function, many complications can occur ${ }^{5}$. The literature has reported plate exposure as the most frequent complication ${ }^{5}$. The possible factors involved are both contracture and a tenuous vascular supply of soft tissues overlying the plate. Scar contracture produces a retraction toward the side of the dead space created under the plate. Consequently, the plate continues to exercise pressure on the overlying tissues and results in necrosis and exposure over time ${ }^{5}$. Furthermore, the disconnection of the masticatory muscles can be the cause of intraoral plate exposure, especially in patients who underwent anterior mandibulectomy ${ }^{7}$. For this reason, anterior mandibular defects are usually more complex to reconstruct without bone free flaps, compared to lateral defects ${ }^{4}$.

The surgical algorithm and procedure described in the present study consisted in the creation of a customised bridging mandibular prosthesis, based on patient's specific mandibular prototyping of the native mandible. Computer-assisted design and computer-assisted manufacturing have recently become a significant improvement in mandibular reconstructions ${ }^{13}{ }^{14}$. This approach is very useful when secondary reconstruction is performed. In fact, in these cases the local clinical conditions and the quality of the remaining bone are usually not adequate to perform a new plate fixation. Printing titanium CAD/CAM prosthesis helped us to have larger anchorage surface on the bone and larger bone contact. This can reduce the problem relative to plate fracture for inadequate biomechanical forces on the hardware surface.

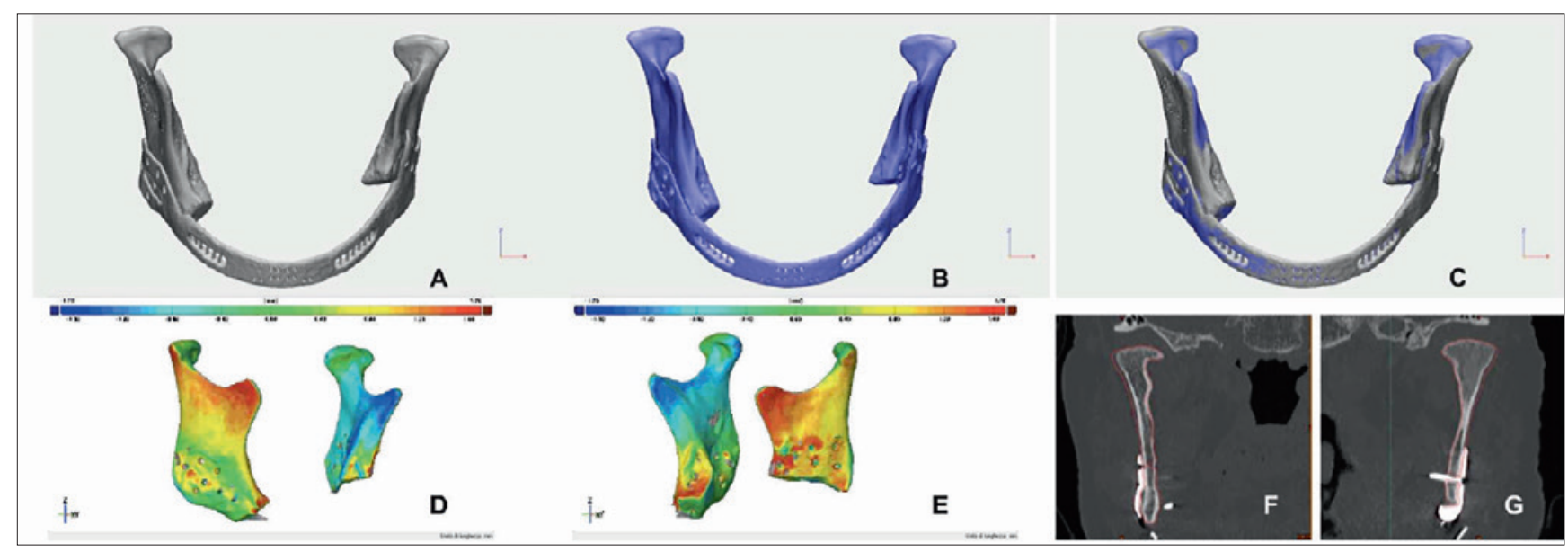

Fig. 10. Post-operative accuracy evaluation. A: virtual planning; B: post-operative 3D CT-scan; C: superimposition of the virtual planning with the postoperative CT scan. D-E: error grade colour map. It provides a direct impression of the concordance between the pre-operative design and the post-operative position of the mandibular rami. F-G: CT-scan concordance between virtual planning (red line) and post-operative situation (coronal view). 
Furthermore, virtual planning is of crucial importance for the setting of the new screw holes in the right points of the bone, making this procedure surgically safer. Moreover, this planning permits to have at least 3 points of screw fixation to achieve the best obtainable stability ${ }^{15}$.

Biomechanically, the load is efficiently and largely distributed on the new plate, since it has a larger surface than a standard reconstructive plate. In addition, bone-titanium surface contact was realised in order to achieve the best fitting of the CBMP on the mandible.

To reduce the risk of oral exposure, the prosthesis was designed to be more likely positioned along the lingual cortex of the native mandibular bone, thus reducing tension on soft tissues.

Another new feature that we added on the CBMP were the holes for muscle insertion. This way we guaranteed both a better recovery of mandibular movements and the physiological balance between elevator and depressor muscles. By printing the prosthesis based on the native mandible, we had good aesthetic results for the patient, giving a natural contouring to the inferior mandibular region.

Potential disadvantages of this technique include the cost of designing and prototyping the device. This aspect should be considered in the following way: the effective cost of CBMP should be considered as a tool that can reduce the needing for secondary or tertiary revision procedures for plate dislocation or exposure.

Another disadvantage of the proposed protocol is that it is impossible to obtain dental rehabilitation: due to lack of bone graft, it is not possible to perform implant-supported rehabilitation. In the described case, the patient was edentulous before surgery.

In conclusion, although longer follow-up is needed to evaluate long-term results, CBMP may be a viable method for mandibular reconstruction in patients who cannot undergo microvascular reconstruction with bony free flap.

\section{References}

1 Lonie S, Herle P, Paddle A, et al. Mandibular reconstruction: meta-analysis of iliac-versus fibula-free flaps. ANZ J Surg 2016;86:337-42.

2 Kokosis G, Schmitz R, Powers DB, et al. Mandibular reconstruction using the free vascularized fibula graft: an overview of different modifications. Arch Plast Surg 2016;43:3-9.

3 Moubayed SP, L'Heureux-Lebeau B, Christopoulos A, et al. Osteocutaneous free flaps for mandibular reconstruction: systematic review of their frequency of use and a preliminary quality of life comparison. J Laryngol Otol 2014;128:1034-43.

4 Poli T, Ferrari S, Bianchi B, et al. Primary oromandibular reconstruction using free flaps and thorp plates in cancer patients: a 5-year experience. Head Neck 2003;25:15-23.

5 Okura M, Isomura ET, Iida S, et al. Long-term outcome and factors influencing bridging plates for mandibular reconstruction. Oral Oncol 2005;41:791-8.

6 Bedogni A, Bettini G, Ferronato G, et al. Replacement of fractured reconstruction plate with customized mandible implant: a novel technique. Laryngoscope 2014;124:401-4.

7 Fanzio PM, Chang KP, Chen HH, et al. Plate exposure after anterolateral thigh free-flap reconstruction in head and neck cancer patients with composite mandibular defects. Ann Surg Oncol 2015;22:3055-60.

8 Klotch DW, Prein J. Mandibular reconstruction using AO plates. Am J Surg 1987;154:384-8.

9 Kudo K, Shoji M, Yokota M, et al. Evaluation of mandibular reconstruction techniques following resection of malignant tumors in the oral region. J Oral Maxillofac Surg 1992;50:14-21.

10 Tsuchiya S, Nakatsuka T, Sakuraba M, et al. Clinical factors associated with postoperative complications and the functional outcome in mandibular reconstruction. Microsurgery 2013;33:337-41.

11 Zavattero E, Fasolis M, Garzino-Demo P, et al. Evaluation of plate-related complications and efficacy in fibula free flap mandibular reconstruction. J Craniofac Surg 2014;25:397-9.

12 Leiggener C, Messo E, Thor A, et al. A selective laser sintering guide for transferring a virtual plan to real time surgery in composite mandibular reconstruction with free fibula osseous flaps. Int J Oral Maxillofac Surg 2009;38:187e192.

13 Tarsitano A, Mazzoni S, Cipriani R, et al. The CAD-CAM technique for mandibular reconstruction: an 18 patients oncological case-series. J Craniomaxillofac Surg 2014;42:1460-4.

14 Tarsitano A, Del Corso G, Ciocca L, et al. Mandibular reconstructions using computer-aided design/computer-aided manufacturing: a systematic review of a defect-based reconstructive algorithm. J Craniomaxillofac Surg 2015;43:1785-91.

15 Kimura A, Nagasao T, Kaneko T, et al. Adaquate fixation of plates for stability during mandibular reconstruction. J Craniomaxillofac Surg 2006;34:193-200.

Received: May 25, 2016 - Accepted: June 19, 2016

Address for correspondence: Achille Tarsitano, Maxillofacial Surgery Unit, S. Orsola-Malpighi Hospital, Department of Biomedical and Neuromotor Sciences, Alma Mater Studiorum University of Bologna, via G. Massarenti 9, 40100 Bologna, Italy. E-mail: achille.tarsitano2@unibo.it; achilletarsitano@gmail.com 